ReVISTA BRASILEIRA DE ANÁlise do COMPORTAMENTO / BRAZILIAN JOURNAL OF BEHAVIOR ANALYSIS, 2018, VOL. 14, N². 2, $136-143$.

\title{
O ESTUDO DA PUNIÇÃO COM O JATO DE AR QUENTE: EFEITO DE DIFERENTES HISTÓRIAS DE REFORÇAMENTO
}

\section{THE STUDY OF PUNISHMENT WITH HOT BLAST AIR: EFFECT OF DIFFERENT REINFORCEMENT HISTORIES}

\author{
MARCUS Bentes de CARVALHo NETO - ORCID 0000-0001-9550-409X \\ UNIVERSIDADE FEDERAL DO PARÁ, BRASIL
}

PRISCILA GISELLI SILVA MAGALHÃES - ORCID 0000-0001-7209-2985

UNIVERSIDADE FEDERAL DO PARÁ, BRASIL

\author{
BRUNA COLOMBO DOS SANTOS - ORCID 0000-0002-7574-1714

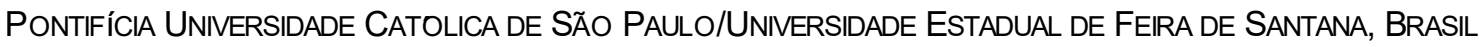

PAulo CÉSAR MORALES MAYER - ORCID 0000-0001-9484-2118

UNIVERSIDADE CEUMA, BRASIL

\begin{abstract}
RESUMO
Uma das variáveis que tem influência sobre os efeitos de contingências punitivas é a história de reforçamento. Estudos com choque elétrico demonstram que esquemas de reforçamento contínuo produzem menor resistência à punição, do que esquemas de reforçamento intermitentes. $\mathrm{O}$ objetivo desse estudo foi verificar o efeito de um estímulo punidor alternativo - o jato de ar quente (JAQ) - apresentado de acordo com um esquema FR1, sobre a frequência de respostas operantes fortalecidas por diferentes histórias de reforçamento. Seis ratos foram expostos a diferentes histórias de reforçamento durante oito sessões (reforçamento contínuo, razão variável e intervalo variável). Em seguida, os animais passaram por seis sessões em que cada resposta de pressão à barra produzia um JAQ e uma gota de água. Por fim, os animais passaram por seis sessões de recondicionamento, com os mesmos esquemas utilizados na fase de fortalecimento. Durante as sessões de punição, todos sujeitos, independente da história de reforçamento apresentaram total supressão do responder. Quando a punição foi descontinuada todos sujeitos retornaram aos níveis prévios de resposta compatíveis com suas respectivas histórias de reforçamento, os sujeitos do grupo de reforçamento contínuo, contudo, levaram mais tempo para recuperar as taxas de respostas. Limitações metodológicas que comprometeram as conclusões do estudo são discutidas no intuito de aperfeiçoamento.

Palavras-chave: punição; história de reforçamento; jato de ar quente; controle aversivo.
\end{abstract}

\begin{abstract}
One of the variables that has influence on the effects of punishing contingencies is reinforcement history. Studies with electric shock demonstrated that continuous reinforcement schedules produce less resistance to punishment than intermittent reinforcement schedules. The aim of this study was to verify the effect of an alternative punisher - hot blast air (HBA) - presented continuously and contingent to responses with different reinforcement histories. Six rats were exposed to different histories of reinforcement during eight sessions (continuous reinforcement, variable ratio and variable interval). Then, the animals went through six sessions in which each bar pressing produced an HBA and a drop of water. In the last phase, the animal went through six reconditioning sessions, with the same schedules used in conditioning phase. During punishment, all subjects, regardless of reinforcement history show completely suppression of responding. When punishment was discontinued all subjects returned to the previous response levels compatible with their reinforcement history, the subjects which passed by continuous reinforcement, however, took more time to recover the responses rates. Methodological limitations that compromised the study conclusions are discussed for improvement.

Key words: punishment; reinforcement history; hot blast air; aversive control.

Endereço para correspondência: Rua Augusto Corrêa, 01, Campos Universitário do Guamá, Belém, Pará, Brasil, CEP: 66075110. Telefone: (91) 3201-8542/8476. Email: brucolombodossantos@gmail.com. Trabalho parcialmente financiado pelo CNPq através de bolsa PIBIC/CNPq (concedida ao $2^{\circ}$ autor) e do Edital Universal 2005 (Processo No. 476238/04-8).
\end{abstract}

DOI. $10.18542 /$ rebac.v14i2.7533 
Historicamente, pesquisas básicas sobre punição, com sujeitos não humanos, têm utilizado sistematicamente o choque elétrico como estímulo punidor. O choque apresenta vantagens como ser mensurado precisamente e poder ser apresentado em diferentes intensidades, o que permite uma quantificação precisa dos resultados em relação a cada parâmetro do estímulo manipulado, replicação dos estudos em diferentes laboratórios e uma comparação mais direta entre estudos (Azrin \& Holz, 1966; Catania, 1999).

Entretanto, o uso predominante do choque elétrico tem sido considerado como uma das variáveis responsáveis pelo declínio das pesquisas em controle aversivo, em relação a outras áreas em Análise do Comportamento (Baron, 1991; Catania, 2008). A utilização do choque elétrico em pesquisas básicas produz algumas desvantagens de ordem metodológica e ética, tais como alterações fisiológicas ou motoras que podem interferir na ocorrência da resposta operante estudada e o sofrimento infligido ao animal (Catania, 2008), além de ser questionável se os resultados dos estudos com choque são generalizáveis para outros estímulos de natureza e efeitos fisiológicos distintos (Dinsmoor, 1998).

Alguns estudos testaram efeitos punitivos de estímulos alternativos ao choque elétrico, como por exemplo: barulho intenso, luz brilhante e timeout (e.g., Holz \& Azrin, 1962; Barker et al., 2010). Nessa linha de investigação, o jato de ar quente (JAQ) tem sido testado em diversos procedimentos aversivos, incluindo contingências punitivas. O JAQ se mostrou um estímulo punidor eficaz em esquemas de punição contínua, intermitente e em punição de respostas consumatórias (Carvalho Neto, Maestri, \& Menezes, 2007; Carvalho Neto et al., 2005; Mayer, Silva, \& Carvalho Neto, 2015).

Na pesquisa sobre punição utilizando choque elétrico, uma das variáveis que foi investigada como tendo efeito potencial sobre contingências punitivas é a história de reforçamento da resposta punida (Azrin \& Holz, 1966; Lerman $\&$ Vorndran, 2002). Hineline (1984) argumenta que uma das justificativas utilizadas para manutenção do controle aversivo como um domínio separado dentro da análise do comportamento, tem sido, justamente, a diferença entre reforçamento e punição. Um dos argumentos é que a punição seria um processo mais complexo, por envolver uma contingência que é aplicada sobre outra contingência (de reforçamento). Logo, os efeitos da punição sobre o comportamento dependeriam além do estímulo punitivo, sua intensidade e esquema de apresentação, da interação que a contingência punitiva tem com a contingência de reforçamento em vigor que mantém o operante, assim como de sua história de reforçamento.

Uma das características da contingência de reforçamento é o modo como o estímulo reforçador é apresentado. Esse estímulo pode ser apresentado de forma contínua ou intermitente, utilizando como critérios: tempo (intervalo) ou número de respostas (razão) (Catania, 1999). O esquema de reforçamento utilizado produz diferenças com relação à resistência do responder à punição. Esquemas de reforçamento contínuo produzem uma menor resistência à punição do que esquemas de reforçamento intermitentes. Dessa forma, o responder seria mais facilmente enfraquecido pela punição após uma história de reforçamento para cada resposta (Azrin \& Holz, 1966).

Azrin (1960), Filby e Appel (1966), Church e Raymond (1967) e Powell (1970) inseriram esquemas de punição (contínua/intermitente) sobre uma linha de base mantida por um esquema de reforçamento intermitente, tanto de intervalo variável (VI) quanto de razão variável (VR). Azrin (1960) expôs pombos a um esquema de reforçamento em intervalo variável de 1 ou 3 minutos (VI 1-min ou VI 3-min, respectivamente). Após o critério de estabilidade ter sido atingido, foram apresentadas diferentes intensidades e durações de choque elétrico (e.g., 20 volts, 40 volts, 50 volts e 130 volts; com durações entre 30 e 100 milissegundos) contingente a toda resposta de bicar (FR 1). De maneira geral, os dados demonstraram que a taxa de respostas diminuiu com o aumento progressivo da intensidade de choque elétrico, não havendo diferenças significativas no responder dos sujeitos com relação ao esquema de intervalo.

Filby e Appel (1966) expuseram três grupos de ratos a três intervalos diferentes de VI (VI 30-seg, VI 1-min e VI 3min) para fortalecimento da resposta de pressão à barra. Em seguida, cada grupo teve o esquema de VI para reforçamento mantido e, as respostas passaram a ser, também, punidas em esquema de intervalo variável (VI 30-seg, VI 1-min e VI 3min). A intensidade do choque variou entre 0,2 e 1,3 miliamperes (mA). A intensidade do choque pareceu ser a variável mais relevante para supressão de respostas durante à punição, pois ocorreu supressão completa, com pouca ou nenhuma recuperação, apenas em intensidades mais elevadas (a partir de 0,6 mA). Todavia, o intervalo de VI para reforçamento exerceu influência sobre a recuperação de respostas após a retirada do choque. A recuperação das respostas foi mais rápida para o grupo de menor intensidade $(0,2 \mathrm{~mA})$ e menor valor do intervalo variável (VI 30-seg).

Church e Raymond (1967) utilizaram um esquema de intervalo variável de 30 segundos e cinco minutos (VI 30-seg e VI 5-min, respectivamente) para manutenção da resposta de pressão à barra em dois grupos de ratos. Em seguida, metade dos animais de cada grupo também foi exposto a um esquema de punição em intervalo variável de dois minutos (VI 2-min), com intensidade de $0,15 \mathrm{~mA}$. A outra metade permaneceu sem ser exposta à punição, como controle. Os dados demonstraram que a efetividade do choque em suprimir as respostas de pressão à barra foi inversamente relacionada à taxa de reforçamento positivo.

Powell (1970, experimento 1) comparou os efeitos da intensidade do choque sob resposta fortalecidas em um esquema múltiplo razão variável e intervalo variável. Pombos foram expostos a um esquema múltiplo progressivo VR $100 \mathrm{VI}$ 1 minuto. Em seguida, um choque de 0,20 segundos de duração era apresentado contingente à décima resposta em cada esquema (punição em FR10). A intensidade do choque inicial foi 1,30 mA, tendo gradualmente aumentado até $20 \mathrm{~mA}$. Por 
fim, os animais foram expostos a uma fase onde o choque foi gradualmente retirado. Os resultados demonstraram que, conforme a intensidade do choque foi aumentada, o responder mantido em VR foi suprimido mais completamente do que o responder mantido em VI. Com relação à recuperação de respostas, houve maior recuperação inicial para respostas mantidas em VI.

Os dados de Filby e Appel (1966), Church e Raymond (1967) e Powell (1970) mostram que o esquema de reforçamento positivo intermitente, seja ele variável ou em razão, foi uma variável relevante para: (a) a supressão de respostas durante a punição - o responder mantido em esquema de intervalo variável com maior taxa de reforçamento positivo é menos sensível ${ }^{1}$ à punição e, se comparados VR e VI, o responder mantido em VR é mais sensível à punição; (b) recuperação de respostas após a retirada do choque - parece haver maior recuperação para o responder mantido por esquema de intervalo variável

Levando em consideração os dados sobre o efeito de diferentes histórias de reforçamento sobre supressão e recuperação de respostas após a punição, utilizando como estímulo o choque elétrico, indaga-se se resultados similares poderiam ser produzidos utilizando um tipo de estímulo punidor alternativo. Portanto, o objetivo deste estudo foi verificar os efeitos da apresentação do JAQ, de acordo com um esquema FR 1, sobre a frequência de respostas operantes fortalecidas por diferentes histórias de reforçamento (contínuo, intervalo variável e razão variável)

\section{MÉTODO}

\section{Sujeitos}

três fêmeas), da espécie Rattus norvegicus, experimentalmente ingênuos, provenientes do Biotério do Instituto Evandro Chagas. As fêmeas começaram o experimento com aproximadamente 90 dias de idade e os machos com aproximadamente 124 dias de idade. O regime alimentar para todos os sujeitos foi ad libitum. Durante o experimento, os animais eram privados de água por 48 horas, tendo acesso livre durante 30 minutos após cada sessão. Os ratos eram mantidos em pares em suas gaiolas viveiro, forradas com maravalha, trocada a cada quatro dias, em condições estáveis de umidade e temperatura e controle natural do ciclo claro-escuro (12h-12h).

\section{Equipamento e materiais}

Uma caixa de condicionamento operante fabricada originalmente pela FUNBEC, medindo $20 \mathrm{~cm}$ de comprimento, $25 \mathrm{~cm}$ de largura e $25 \mathrm{~cm}$ de altura, com paredes em alumínio, o teto e uma das paredes laterais eram em acrílico transparente, permitindo a observação do sujeito.
A caixa foi alterada para receber um secador de cabelos na sua parte superior: foi aberto um quadrado de $6 \mathrm{~cm}$ no teto sobre a barra, sobre este havia uma tela de arame (com quadrados de $1 / 2 \mathrm{~cm}$ ) que separava o interior da caixa de um secador de cabelo acoplado sobre a caixa de condicionamento por um suporte de papelão (ver Figura 1). O piso da caixa era composto de barras metálicas cilíndricas, abaixo desta havia uma bandeja permitindo o recolhimento de dejetos. Na parte interna da caixa, na parede lateral direita ficava uma barra metálica cilíndrica, na horizontal, com 4 $\mathrm{cm}$ de comprimento e $1 \mathrm{~cm}$ de diâmetro. Abaixo da barra havia um bebedouro que fornecia através de um pequeno copo uma gota de água quando acionado (aproximadamente $20 \mathrm{ml}$ ). O acionamento do bebedouro e a contagem de pressões à barra eram realizados através de uma caixacontrole operada pelo experimentador.

Foram utilizados dois cronômetros digitais, possuindo funções de relógio e cronômetro, folhas de registro (uma para cada sessão), uma prancheta e caneta.

Para a produção do jato de ar quente, foi utilizado um secador de cabelos com potência de 1600 Watts, fabricação REVLON, modelo RV 429AB. Na intensidade máxima (aqui adotada) produzia, ao final de 5 segundos, um aumento de 4 graus Celsius em relação à temperatura ambiente em um raio de $10 \mathrm{~cm}$, com a pressão de 216,5 dyn/cm2 e um nível de ruído de $85 \mathrm{db}$. O seu acionamento era executado manualmente pelo experimentador através de um interruptor adaptado ao cabo de força do secador.

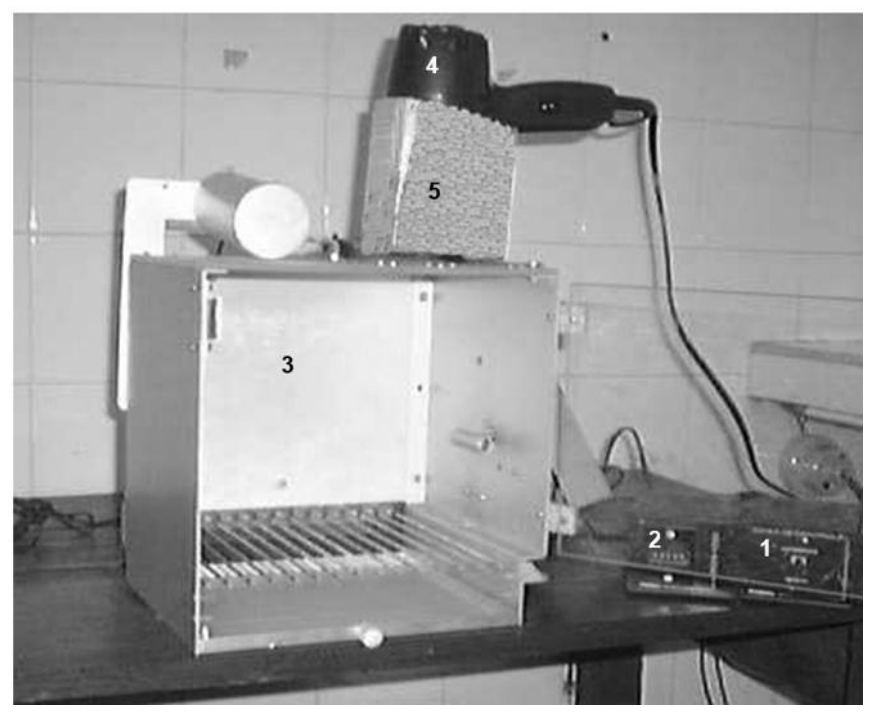

Figura 1. Caixa de condicionamento operante adaptada composta por: (1) Caixa de controle do bebedouro; (2) Contador numérico; (3) Câmara Experimental; (4) Secador de cabelo; (5) Suporte.

\footnotetext{
${ }^{1}$ Sensibilidade, nesse texto, diz respeito a uma mudança comportamental ordenada e replicável, produzida por uma manipulação experimental (Madden, Chase, \& Joyce, 1998)
} 
Tabela 1

Fases do experimento 1 para os sujeitos que passaram pelas histórias CRF, VR5 e VI5".

\begin{tabular}{|c|c|c|c|c|c|}
\hline História & $1^{\mathrm{a}}$ Fase & $2^{\mathrm{a}}$ Fase & $3^{a}$ Fase & $4^{a}$ Fase & $5^{\text {a }}$ Fase \\
\hline CRF & $\begin{array}{l}\text { Nível Operante: } \\
1 \text { sessão }\end{array}$ & $\begin{array}{c}\text { Modelagem da } \\
\text { RPB: } \\
1 \text { sessão }\end{array}$ & $\begin{array}{l}\text { Água em } \\
\text { CRF: } \\
8 \text { sessões }\end{array}$ & $\begin{array}{c}\text { Ar Quente em } \\
\text { CRF } \\
+ \\
\text { Água em CRF } \\
\text { (Contínuo): } \\
6 \text { sessões }\end{array}$ & $\begin{array}{c}\text { Água em CRF: } \\
6 \text { sessões }\end{array}$ \\
\hline VR5 & $\begin{array}{c}\text { Nível Operante: } \\
1 \text { sessão }\end{array}$ & $\begin{array}{c}\text { Modelagem da } \\
\text { RPB: } \\
\text { 1 sessão }\end{array}$ & $\begin{array}{l}\text { Água em } \\
\text { VR5: } \\
8 \text { sessões }\end{array}$ & $\begin{array}{c}\text { Ar Quente em } \\
\text { CRF } \\
+ \\
\text { Água em CRF } \\
\text { (Contínuo): } \\
6 \text { sessões }\end{array}$ & $\begin{array}{c}\text { Água em VR5: } \\
6 \text { sessões }\end{array}$ \\
\hline VI5" & $\begin{array}{c}\text { Nível Operante: } \\
1 \text { sessão }\end{array}$ & $\begin{array}{c}\text { Modelagem da } \\
\text { RPB: } \\
1 \text { sessão }\end{array}$ & $\begin{array}{l}\text { Água em } \\
\text { VI5": } \\
8 \text { sessões }\end{array}$ & $\begin{array}{c}\text { Ar Quente em } \\
\text { CRF } \\
+ \\
\text { Água em CRF } \\
\text { (Contínuo): } \\
6 \text { sessões }\end{array}$ & $\begin{array}{c}\text { Água em VI5": } \\
6 \text { sessões }\end{array}$ \\
\hline
\end{tabular}

\section{Procedimento}

Cada sessão do experimento teve duração de 60 minutos. Previamente a cada sessão, os sujeitos eram privados de água por 48 horas $^{2}$. Durante a primeira sessão os sujeitos foram introduzidos na caixa sem qualquer manipulação experimental para aclimatação. $\mathrm{Na}$ segunda sessão foi realizado o treino de pressão a barra por reforçamento diferencial de aproximações sucessivas, acionando-se o bebedouro sempre que respostas em direção à resposta alvo (pressão a barra) ocorriam. Respostas de pressão a barra eram reforçadas continuamente (CRF). Após o treino de pressão à barra, cada dois sujeitos foram expostos a contingências de reforçamento distintas: reforçamento contínuo (CRF), razão variável cinco (VR-5) e intervalo variável cinco segundos (VI-5s).

Os sujeitos foram submetidos inicialmente a oito sessões de treino em diferentes esquemas de reforço (fortalecimento). Para os sujeitos em CRF cada pressão a barra produzia uma gota de água no bebedouro. Para os sujeitos em VR5 operava um esquema de razão variável no qual uma gota de água era liberada em média a cada cinco pressões a barra (critério variando de duas a oito respostas). Já para os sujeitos em VI5 s, operava um esquema de intervalo variável, no qual uma gota de água era liberada a cada cinco segundos, desde que pelo menos uma resposta de pressão a barra ocorresse após transcorrido o intervalo programado (foram utilizados 5 intervalos variando de dois a

\footnotetext{
${ }^{2} \mathrm{O}$ estudo ocorreu antes da existência de comitê de ética na Universidade Federal do Pará (UFPA). Todavia o primeiro autor, na época, era membro afiliado do COBEA (Colégio Brasileiro de Experimental Animal) e garante que todos os procedimentos éticos foram seguidos.
}

oito segundos). A gota de água, uma vez produzida, ficava à disposição do rato para consumo, sem restrição de tempo para consumo.

$\mathrm{Na}$ fase seguinte, todos os animais foram submetidos a uma contingência de punição em razão fixa (FR1), no qual toda pressão a barra era seguida pela a liberação de um JAQ, na intensidade máxima por 5 segundos (punição). Sobreposto a esse esquema toda pressão a barra produzia, também, uma gota de água (CRF). Essa fase durou seis sessões de 60 minutos. Na última fase do estudo (recondicionamento) cada sujeito foi submetido a seis sessões de fortalecimento de acordo com a sua história experimental (CRF, VR-5, VI-5") do mesmo modo que na fase anterior à punição. $\mathrm{O}$ critério de encerramento de cada sessão foi tempo (60 minutos).

A Tabela 1 resume as etapas do procedimento experimental descrito anteriormente.

\section{RESULTADOS}

A Figura 2 mostra os dados individuais para os sujeitos durante cada sessão, nas etapas de fortalecimento, punição e recondicionamento.

Conforme a Figura 2 (dados a esquerda), a taxa de respostas nas sessões de fortalecimento foi menor para os sujeitos que passaram pelo CRF em comparação com os sujeitos que passaram pelo VI e VR. Comparando os sujeitos que passaram pelos esquemas VR e VI, observa-se que a taxa de respostas foi maior para os dois sujeitos em VR, em praticamente todas as sessões, com exceção das sessões seis e sete para o sujeito S1 que passou por VI. 
Ainda na Figura 2 (dados da posição central), é possível observar que o jato de ar quente suprimiu o responder, independentemente da história prévia de fortalecimento. Todos os sujeitos tiveram as taxas de respostas reduzidas virtualmente para zero, com exceção do sujeito S2, que passou pela história em CRF, na última sessão da fase "punição" (14 respostas).
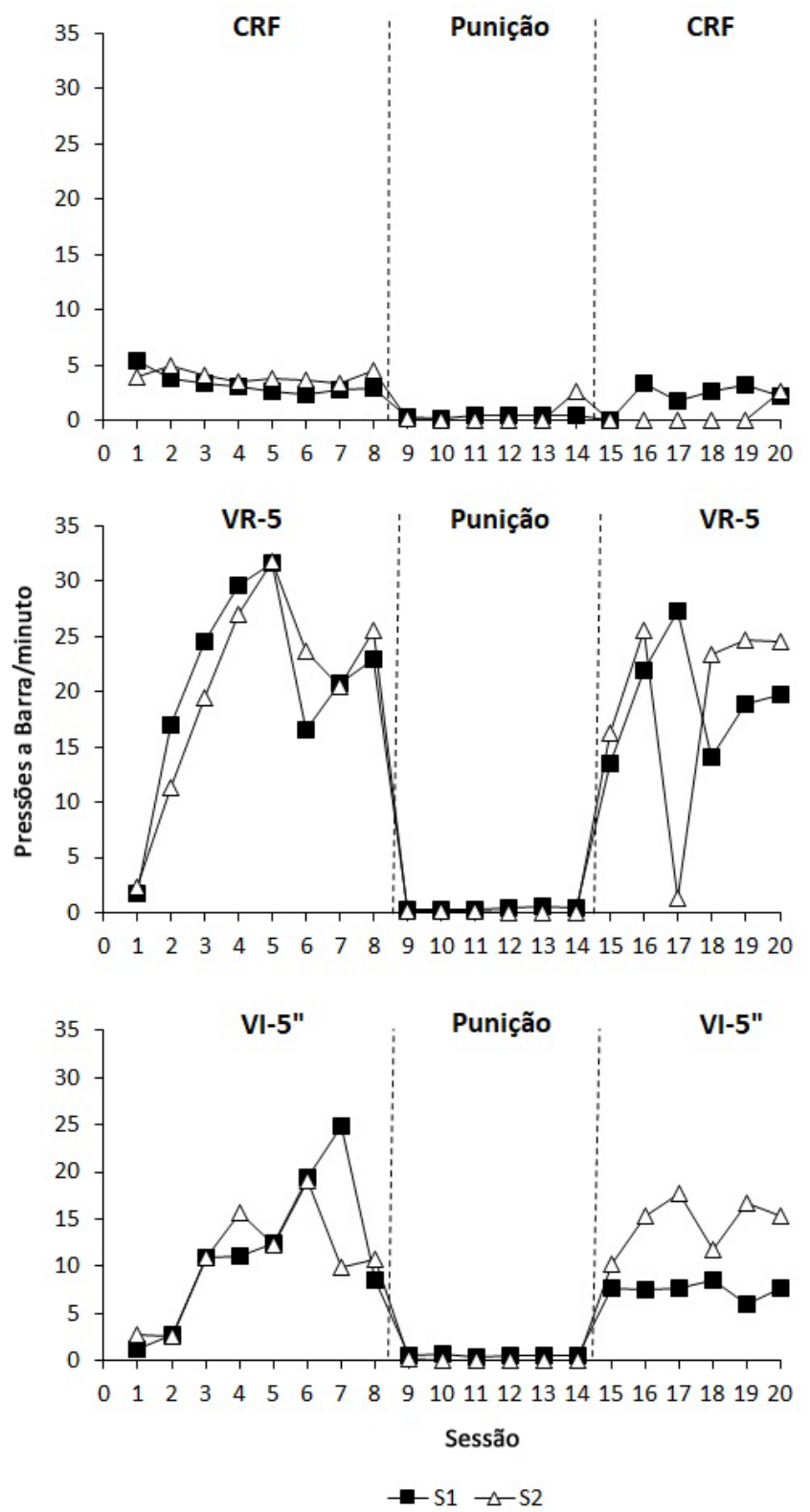

Figura 2. Taxa de respostas de pressão a barra por sessão para os sujeitos que passaram por treino em CRF, VR e VI durante as fases: fortalecimento, punição e recondicionamento.

Durante o recondicionamento (Figura 2, dados à direita), é possível observar que todos sujeitos, independente da história de reforço voltaram a responder quando a punição foi descontinuada. Os sujeitos expostos a histórias de reforçamento intermitente voltaram a pressionar a barra já na primeira sessão de recondicionamento, o sujeito S1 do grupo de reforçamento contínuo retornou a responder na segunda sessão e o S2 na sexta sessão. O padrão da curva de respostas foi similar ao da fase de fortalecimento para todos os sujeitos, exceto para o sujeito S1 que passou pelo esquema VI, o qual apresentou um padrão de respostas mais homogêneo entre sessões, na fase de recondicionamento, em comparação com o padrão oscilante da fase de fortalecimento. Os sujeitos cuja história de reforçamento foi intervalo variável apresentaram, na primeira sessão após a punição ser descontinuada, taxas de respostas similares à da última sessão de fortalecimento. Os sujeitos cuja história de reforçamento foi CRF ou VR-5, apresentaram taxas de respostas menores nessa primeira sessão de que na última sessão de fortalecimento.

\section{DISCUSSÃO}

Os dados obtidos demonstraram que os sujeitos, independente das histórias de reforçamento, apresentaram supressão comportamental similar enquanto a punição estava em vigor. Além disso, todos retomaram o responder em taxas semelhantes aos seus níveis prévios após a punição ser descontinuada. Tais constatações, contudo, não devem ser compreendidas como indicativo de que a história de reforçamento não seja uma variável relevante na determinação dos efeitos da punição. A relevância de tal variável, consistentemente identificada em diferentes revisões (e.g. Azrin \& Holz, 1966; Church, 1963; Morse \& Kelleher, 1977), sugere uma análise crítica dos métodos utilizados no presente estudo e suas potenciais limitações.

A relação entre os efeitos da punição (i.e., supressão e posterior recuperação do responder) e a história de reforçamento é um tópico complexo que envolve um amplo conjunto de variáveis. Dentre elas, a densidade e a probabilidade de reforço e da punição, a natureza e intensidade do estímulo punidor e a duração das fases de treino e de punição. Dentre estas, a intensidade do estímulo aversivo parece ser a variável mais determinante quando elevada (Appel \& Peterson, 1965; Azrin, 1960; Boe \& Church, 1967).

A elevada supressão produzida pelo JAQ, assim como ausência de recuperação do responder durante a fase de punição, atestam a alta intensidade do estímulo utilizado. O efeito piso da supressão produzida pelo JAQ não permitiu uma comparação refinada entre as diferentes histórias de reforçamento. Nesse sentido, o presente estudo corrobora a constatação de que a elevada intensidade do estímulo punidor é uma variável suficiente para a determinar a supressão comportamental, sobrepondo-se aos efeitos da história de reforçamento. Contudo, tais dados apontam para a inadequação do JAQ para o teste de algumas variáveis mais sutis da punição quando administrado nas dimensões tipicamente utilizadas até o momento. 
Uma segunda limitação foi a mudança da contingência de reforçamento positivo na fase de punição para os grupos de reforçamento intermitente. $\mathrm{O}$ uso de reforçamento contínuo durante a punição foi uma tentativa de manter constante a taxa de reforçamento, para que esta não interferisse nos efeitos supressivos da punição. Entretanto, tal controle pode interferir diretamente nos efeitos da punição, pois além do estímulo punidor há um aumento na densidade de reforço, em relação à fase de treino, o que pode reduzir a eficácia do estímulo punidor (Morse \& Kelleher, 1977). Tal complicação não foi observada no presente estudo, possivelmente devido à elevada intensidade do estímulo punidor, conforme anteriormente apontado. Todavia, sugere-se que em estudos posteriores, durante a punição, seja mantido o mesmo esquema de reforçamento do treino (salvo em situações nas quais a mudança do esquema de reforçamento seja a variável independente testada, variável pouco investigada em estudos de punição).

Ainda como limitação metodológica, aponta-se o número reduzido de sujeitos, não apenas por se tratar de um estudo no qual foi estabelecida uma comparação entre sujeitos, mas devido a possibilidade de diferenças individuais na sensibilidade a eventos aversivos (Church, 1963). Em todas as condições foi possível observar uma diferença que variou de 11 (sujeitos que passaram por VI) a $20 \%$ (sujeitos que passaram por VR) no número de respostas emitidas durante a punição. Assim, para uma análise entre sujeitos adequada, a utilização de um número maior de sujeitos é fundamental para diluir as diferenças individuais permitindo uma melhor avaliação das variáveis independentes testadas.

Uma alternativa ao aumento do número de sujeito é prescindir da análise de grupos, realizando o teste do efeito de diferentes histórias de reforçamento em esquemas múltiplos, nos quais, o mesmo sujeito é submetido a diferentes histórias de reforçamento intercaladas com punição. Nesse caso, sugere-se o controle de ordem de apresentação das diferentes histórias de reforçamento, uma vez que a própria extensão da história de reforçamento (Azrin \& Holz, 1966), sua alternância (Morse \& Kelleher, 1977) e a recorrente reintrodução da contingência de punição (Rachlin, 1966) são variáveis que podem afetar a efetividade da punição.

Apesar de não se poder atribuir à história de reforçamento a diferença observada na recuperação do responder, em função das limitações já apontadas, ainda assim é possível uma análise da recuperação do responder no presente estudo.

Em conformidade com a literatura com choque elétrico, todos os sujeitos voltaram a responder com taxas de respostas similares ao período de fortalecimento pré-punição (Appel \& Peterson, 1965; Azrin \& Holz, 1966, Catania, 1999). Contudo, esse retorno não ocorreu de forma homogênea. Os sujeitos com história em CRF levaram mais tempo para voltar a responder (1 sessão para o S1 e cinco sessões para o S2), enquanto para os demais sujeitos essa recuperação já ocorreu na primeira sessão em que a punição foi descontinuada.

Apesar dessa constatação estar em conformidade com a afirmação de que uma história de reforço contínuo seria menos resistente à punição (Azrin \& Holz, 1966), uma possível explicação para essa diferença seria o efeito discriminativo das fases do experimento. Como na fase de punição a contingência de reforço era contínua (CRF) não houve discrepância das condições de reforçamento para o grupo CRF nas três fases. Contudo, para os demais sujeitos, pressões a barra que não produziam reforço nem punição (devido a intermitência do reforço) eram mais similares à fase de fortalecimento do que a de punição, podendo ter facilitado a discriminação da mudança de fase (ausência de punição).

Uma questão que chama a atenção durante a fase de recondicionamento foi a ausência de taxas de respostas superiores às do período de fortalecimento. Azrin e Holz (1966) descrevem como uma característica geral do processo de punição um período inicial de aumento da taxa de respostas logo após a punição ser descontinuada, sendo essas taxas maiores do que as do período pré-punição. Os autores denominaram de "contraste" esse aumento inicial na taxa de respostas.

A literatura a respeito do contraste após punição é escassa, não homogênea e aparentemente secundária a outros parâmetros dos estudos. Doleys e Davidson (1974), por exemplo, observaram contraste nos dados de apenas um dos cinco sujeitos pesquisados. Doleys e Davidson (1976), utilizando procedimentos muito semelhantes ao estudo de 1974, observaram contraste nos dados dos dois sujeitos do estudo. Appel e Peterson (1965) discorrem que a recuperação da resposta após o período de punição ocorre mesmo quando a supressão do responder é superior a $80 \%$ do responder em linha de base, e que o contraste comportamental ocorre em alguns casos, embora não apontem quais suas variáveis determinantes. Afirmam ainda que, a ausência de recuperação só ocorre para os casos em que foi utilizado um punidor intenso (no caso choque) produzindo supressão completa do responder de modo imediato (com poucas exposições à punição).

No presente estudo, apesar da supressão ter sido próxima a 100\%, mesmo ao longo de seis sessões para praticamente todos os sujeitos, observou-se recuperação do responder em todos os casos (embora em momentos distintos) sem a ocorrência do contraste. Embora não se possa afirmar categoricamente que essa diferença se deva à natureza do estímulo punidor, é possível afirmar que apenas a intensidade do estímulo, assim como a elevada supressão do responder, não são variáveis suficientes para prevenir a recuperação do responder pós-punição e que o processo de recuperação não é tão homogêneo e sistemático como, por vezes, documentado.

A punição é um fenômeno complexo e sutil, do qual infelizmente conhece-se pouco sobre suas características e 
determinantes. A proposta do presente estudo foi a de verificar o efeito de diferentes histórias de reforçamento sobre a supressão e a recuperação do responder submetido à uma contingência de punição. Os resultados em relação à essa questão foram inconclusivos, contudo, considera-se que o presente estudo oferece contribuições metodológicas valiosas em relação ao estudo da punição de um modo geral.

Enquanto recomendações para futuros estudos destaca-se: (1) a pertinência de se utilizar um estímulo punidor de intensidade branda à moderada, se o objetivo for a identificação do efeito diferencial de variáveis pontuais em relação à efetividade supressiva da punição; (2) a pertinência de se manter o mais constante possível as condições de treino e de punição, principalmente no que se refere à contingência de fortalecimento que mantém o responder (salvo em casos em que essa seja a variável independente testada); (3) a inclusão de um número adequado de sujeitos para se diluir o efeito de diferenças individuais relacionadas a sensibilidade ao estímulo punidor; (4) na medida do possível, utilizar esquemas de reforçamento e procedimentos que permitam a utilização de delineamentos de sujeito único (controlando a ordem de apresentação da história quando pertinente); (5) a utilização de critérios de estabilidade (e.g., Schoenfeld, Cumming, \& Hearst, 1956) para mudança de fase ao invés de número fixo de sessões, uma vez que variáveis individuais podem exigir maiores exposições de determinados sujeitos à certas contingências.

\section{DECLARAÇÃO DE CONFLITO DE INTERESSES}

Os autores declaram que não há conflito de interesses relativos à publicação do artigo.

\section{CONTRIBUIÇÃO DE CADA AUTOR}

Todos os autores participaram suficientemente do trabalho para tornar pública sua responsabilidade pelo conteúdo. M. B. Carvalho Neto contribuiu na concepção do trabalho, redação, delineamento experimental e financiamento; P. G. Silva Magalhães contribuiu na coleta de dados; B. C. Santos e P. C. Mayer contribuíram para a redação final do trabalho.

\section{DIREITOS AUTORAIS}

Este é um artigo aberto e pode ser reproduzido livremente, distribuído, transmitido ou modificado, por qualquer pessoa desde que usado sem fins comerciais. $\mathrm{O}$ trabalho é disponibilizado sob a licença Creative Commons 4.0 BY-NC.

\section{(cc)) BY-No}

\section{REFERÊNCIAS}

Appel, J. B., \& Peterson, N. J. (1965). Punishment: Effects of shock intensity on response suppression. Psychological Reports, 16(3), 721-730. doi:

10.2466/pr0.1965.16.3.721
Azrin, N. (1960). Effects of punishment intensity during variable-interval reinforcement. Journal of the Experimental Analysis of Behavior, 3(2), 123-142. doi: 10.1901/jeab.1960.3-123

Azrin, N. N., \& Holz, W. C. (1966). Punishment. In W. K. Honig (Eds.), Operant behavior: Areas of research and application (pp. 380-447). Englewood Cliffs: Prentice-Hall.

Barker, D. J., Sanabria, F., Lasswell, A., Thraikill, E. A., Pawlak, A. P., \& Killeen, P. R. (2010). Brief light as apractical aversive stimulus for the albino rat. Behavior and Brain Research, 214(2), 402-408. doi: 10.1016/j.bbr.2010.06.020

Baron, A. (1991). Avoidance and punishment. In I. H. Iversen, \& K. A. Lattal (Eds.), Experimental analysis of behavior Part I (pp. 173-217). Amsterdan: Elsevier

Boe, E. E., \& Church, R. M. (1967). Permanent effects of punishment during extinction. Journal of Comparative and Physiological Psychology, 63(3), 486-492. doi: 10.1037/h0024632

Carvalho Neto, M. B., Maestri, T. C., \& Menezes, E. S. R. (2007). O jato de ar quente como estímulo aversivo: Efeitos supressivos da exposição prolongada em Rattus norvegicus. Acta Comportamentalia, 15(2), 171-190.

Carvalho Neto, M. B., Maestri, T. C., Tobias, G. K. S., Ribeiro, T. C., Coutinho, E. C. N. N., Miccione, M. M., Oliveira, R. C. V., Ferreira, F. S. S., Farias, D. C., \& Moreira, D. (2005). $\mathrm{O}$ jato de ar quente como estímulo punidor em Rattus norvegicus. Psicologia: Teoria e Pesquisa, 21(3), 335-339. doi: 10.1590/S0102-37722005000300010

Catania, A. C. (1999). Aprendizagem: comportamento, linguagem e cognição (4th ed.; D. G. Souza et al., Trad.). Porto Alegre: Artmed.

Catania, A. C. (2008). The Journal of the Experimental Analysis of Behavior at zero, fifty, and one hundred. Journal of the Experimental Analysis of Behavior, 89, 111118. doi: 10.1901/jeab.2008.89-111

Church, R. M. (1963) The varied effects of punishment on behavior. Psychological Review, 70(5), 369-402. doi: 10.1037/h0046499

Church, R. M., \& Raymond, G. (1967). Influence of the schedule of positive reinforcement on punished behavior. Journal of Comparative and Physiological Psychology, 63(2), 329-332. doi: 10.1037/h0024382

Dinsmoor, J. A. (1998). Punishment. In: W. T. O’Donohue (Ed.), Learning and behavior therapy (pp. 188-204). Boston: Allyn \& Bacon.

Doleys, D. M., \& Davidson, R. S. (1974). Repeated suppression and recovery of responding using response-produced electric shock. Psychological Reports, 35(1), 1259-1268. doi: 10.2466/pr0.1974.35.3.1259

Doleys, D. M., \& Davidson, R. S. (1976). Effects of pairing electric shock with reinforcement delivered on a variableinterval schedule. Psychological Reports, 39(2), 483-489. doi: 10.2466/pr0.1976.39.2.483

Filby, Y., \& Appel, J. B. (1966). Variable-interval punishment during variable-interval reinforcement. Journal of the 
Experimental Analysis of Behavior, 9(5), 521-527. doi: 10.1901/jeab.1966.9-521

Hineline P. N. (1984). Aversive control: A separate domain? Journal of the Experimental Analysis of Behavior, 42(3), 495-509. doi: 10.1901/jeab.1984.42-495

Holz, W. C., \& Azrin, N. H. (1962). Recovery during punishment by intense noise. Psychological Reprints, 11(3), 655-657. doi: 10.2466/pr0.1962.11.3.655

Lerman, D. C. \& Vorndran, C. M. (2002). On the status of knowledge for using punishment: Implications for treating behavior disorders. Journal of Applied Behavior Analysis, 35(4), 431-464. doi: 10.1901/jaba.2002.35-431

Madden, G. J., Chase, P. N., \& Joyce, J. H. (1998). Making sense of sensitivity in the human operant literature. The Behavior analyst, 21(1), 1-12. doi: 10.1007/bf03392775

Mayer, P. C. M., Silva, G. F., \& Carvalho Neto, M. B. (2015). Punishment of instrumental and consummatory responses with a hot air blast in rats. Behavior Analysis: Research and Practice, 15(1), 58-64. doi: 10.1037/h0101070

Morse, W. H., \& Kelleher, R. T. (1977). Determinants of reinforcement and punishment. In W. K. Honig \& J. E. R Staddon (Eds.). Handbook of operant behavior (pp. 174200). Englewood Cliffs, NJ: Prentice-Hall, 174-200.

Powell, R. W. (1970). The effect of punishment shock intensity upon responding under multiple schedules. Journal the Experimental Analysis of Behavior, 14(2), 201-211. doi: 10.1901/jeab.1970.14-201

Rachlin, H. (1966). Recovery of responses during mild punishment. Journal of the Experimental Analysis of Behavior, 9(3), 251-263. doi: 10.1901/jeab.1966.9-251

Schoenfeld, W. N., Cumming, W. W., \& Hearst, E. (1956). On the classification of reinforcement schedules. Proceedings of the National Academy of Sciences of the United States of America, 42(8), 563-570. doi: 10.1073/pnas.42.8.563

Submetido em: 26/03/2019 Aceito em: 14/06/2019 